\title{
ANALOG TRACK ANGLE ERROR DISPLAYS IMPROVE SIMULATED GPS APPROACH PERFORMANCE
}

\author{
Charles M. Oman ${ }^{+}$, M. Stephen Huntley, Jr** , \\ and Scott A. M. Rasmussen ${ }^{+}$ \\ + Man Vehicle Laboratory, Department of Aeronautics and Astronautics \\ Massachusetts Institute of Technology, Cambridge, Massachusetts \\ * Cockpit Human Factors Program, DTS-45 \\ Volpe National Transportation Systems Center, Cambridge, Massachusetts
}

\begin{abstract}
Pilots flying non-precision instrument approaches traditionally rely on a course deviation indicator (CDI) analog display of cross track error (XTE) information. The new generation of GPS based area navigation (RNAV) receivers can also compute accurate track angle error (TAE). Does display of supplementary TAE information improve intercept and tracking performance? Six pilots each flew 20 approaches in a light twin simulator to evaluate 3 different TAE/XTE display formats, in comparison to a conventional receiver CDI display and a more centrally located Horizontal Situation Indicator (HSI). Statistically significant performance improvements were seen in several phases of the approach when using the supplementary TAE information. Analog was preferred over numeric format. However, the advantage was offset by the need to widen the pilot's instrument scan to include the receiver display. Pilots found TAE helpful in establishing intercepts and the appropriate wind correction angle. Findings support the recent FAA TSO-C129 requirement that XTE be presented in the pilot's primary field of view, and the recommendation that avionics manufacturers include supplementary analog TAE display capability.
\end{abstract}

\section{INTRODUCTION}

Satellite based navigation systems and a new generation of microprocessor based cockpit avionics are revolutionizing air traffic control world wide. The FAA has established minimum performance and display airworthiness standards for stand alone GPS equipment (RTCA/DO-208 and TSO C-129), and has begun to publish a new class of GPS nonprecision approaches. This initiative is particularly important for the general aviation (GA) community, since instrument approaches to thousands of new airports will eventually be possible. GPS RNAVs have flexible electronic displays, updatable databases, and many more operating modes than traditional VOR, DME, ILS, and ADF equipment. They will make instrument flying easier and safer, provided that the human factors aspects are properly considered at the design stage.

Most GA aircraft instruments are of the traditional "round dial" type, and panel space is limited. GPS receivers typically fit in a radio slot, often located outside of the pilot's primary scan. Due to severe space constraints, the GPS can have only a small front panel display and a limited set of control buttons and knobs. Since cross track error (XTE) information from the GPS functionally replaces that from VOR/LLS, XTE is typically converted to an analog signal, and displayed on a centrally located CDI or HSI, as shown in Fig. 1. Supplementary XTE information may also be shown on the receiver itself, as in Fig. 2.

Maintaining an aircraft on course centerline is a demanding triple integral manual control task, requiring a hierarchical, multiloop control strategy (Clement, et al, 1968). Pilots must monitor roll attitude and heading and partially base

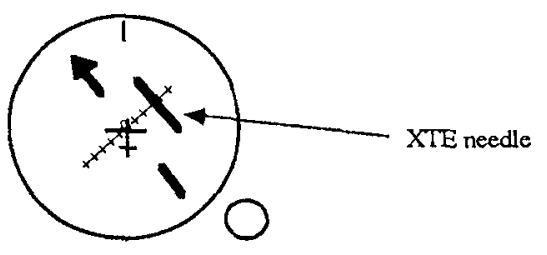

$$
\begin{array}{lll}
\text { NANCY } & \text { ANNIE } \\
\text { XTE } 0.31 & & \\
\text { DIST } 1.2 & & \\
\text { GS } & 124 &
\end{array}
$$

Fig. 1 (Top): Analog XTE on a HSI. (Bottom): Alphanumeric data on nearby GPS receiver display.

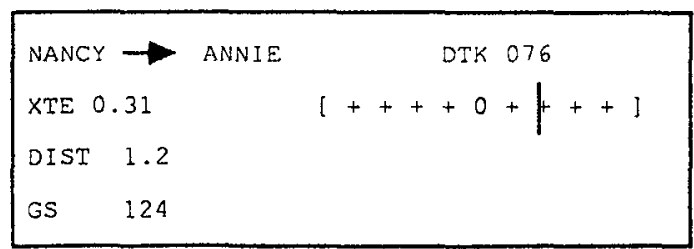

Fig. 2: Analog XTE CDI on GPS receiver display

their control on the rate of change of XTE to avoid large oscillations across the course centerline. The rate of movement of the XTE needle is often not easily perceived. On final approach, XTE needle sensitivity is deliberately increased to help the pilot see its movement and null small 
errors. However, the pilot must then more frequently scan all instruments, reducing the time available to perform other tasks. Inflight studies have demonstrated a direct relationship between CDI sensitivity and pilot workload and an inverse relationship with XTE during non-precision approaches (Huntley, et al, 1991).

GPS RNAV systems can compute "track angle error" (TAE), the difference between the desired track and the actual track (Fig. 3). TAE is mathematically proportional to the rate of change of XTE, the important manual control variable. In principle, if TAE is available, the pilot would not have to monitor the XTE needle as closely to judge its rate of movement. If the aircraft is significantly off course, TAE can be used to establish an appropriate intercept angle. The pilot reduces TAE to zero as the aircraft approaches centerline. Also, it is no longer necessary to use "cut-and-try" techniques to find the heading which eliminates needle "drift" due to crosswinds. By noting the heading at which TAE equals zero, the pilot can immediately establish the appropriate wind correction angle.

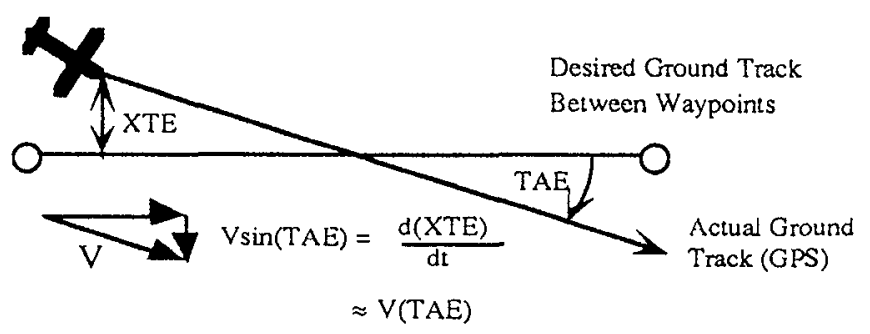

Fig. 3: TAE is proportional to the time derivative of XTE

The FAA's TSO C-129 standard for GPS RNAVs requires at least an alphanumeric display of TAE. The TSO suggests that the use of analog XTE and TAE data integrated "into one display may provide the optimum of situation and control information for the best overall tracking performance". However, the TSO does not specify the graphical format of these displays, in part because pilot performance with supplementary TAE information has never been formally investigated. The goal of this research was to begin this process via simulator experiments, evaluating formats appropriate for the small LCD or CRT displays typically found on the front of many GPS receivers and which conceivably could be implemented merely by a ROM software change. To what extent could such displays allow pilots to quantitatively improve their approach performance, or reduce their workload? A complete report has been published elsewhere (Oman, et al, 1995).

\section{METHODS}

A total of five displays were investigated. The three supplementary TAE display formats evaluated were:

1\&2) Separate TAE and XTE sliding pointer displays ( 2 versions). This format (Fig. 4 , top), originally suggested by FAA's G. Lyddane, added a TAE sliding pointer display beneath a conventional, "fly to" XTE CDI. The pointer was a triangle, located just beneath the XTE needle, and used the same "ten dot" scale. When the triangle was centered, TAE was zero. Full scale pointer deflection was set at $\pm 90^{\circ}$, since this is the maximum useful course intercept angle. Which way should the pointer move? If the triangle moves in the same direction in response as a stick roll command, it is easy to remember, and has the advantage that both the needle and the triangle appear on the same side of the display when converging with course. This display was therefore referred to as "Triangle/Same". However, it is a "fly from" display, whereas the XTE needle above it is "fly to", violating a well known human factors command-response consistency guideline. So a second version was also evaluated, with the opposite sign of the triangle movement. This version (Fig. 4, middle) was referred to as "Triangle/Opposite".

3) An TAE/XTE integrated display. In this format (Fig. 4, bottom), the sliding XTE needle was replaced with a sliding/rotating pointer, whose horizontal position was proportional to XTE as usual and whose tilt angle was equal to the TAE. The display resembled a "mail slot view" of a track-up moving map in an "inside-out", aircraft centered frame of reference, with the arrow corresponding to the desired track. As a result, the pointer tended to move horizontally in the direction it was tilted. This format was referred to as the "Track Vector" display.

Two control conditions were included in which no TAE information explicitly appeared in either numeric or analog format. These were an "HSI" format (Fig. 1) in which XTE was presented along with heading in the pilot's primary instrument scan, with only alphameric information appearing on the receiver, and an "XTE only" receiver display (Fig. 2) which presented analog XTE in the conventional $10 \operatorname{dot}$ CDI format.

The HSI was $70 \mathrm{~cm}$ from the pilot's eye, and $9.5 \mathrm{~cm}$ beneath the attitude indicator. The GPS receiver display was created on a high resolution $L C D$ display, located $35 \mathrm{~cm}$ ( 27 deg.) to the right of the HSI, in a 2 in. by 4 in. area subtending approximately $10^{\circ}$ of horizontal visual angle. A consistent set of generic alphanumeric data was presented on all 5 displays: last and next waypoint, desired track (DTK), numeric XTE, groundspeed (GS), and distance (DIST) to waypoint. Numeric TAE was shown only on TAE displays. In all approaches, the pilot had to monitor DIST, and if a turn at the next waypoint was required, initiate a standard ( $3 \mathrm{deg} / \mathrm{sec}$ ) turn at the appropriate point to intercept the next leg. Waypoints automatically sequenced when the aircraft crossed a line bisecting the angle between the inbound and outbound legs.

Experiments were performed in a fixed base Frasca 242 simulator, using a flight model resembling a Piper Aztec. Patchy, moderate-to-severe turbulence (Jansen, 1981) was added in the attitude axes, requiring the pilot to closely monitor the attitude indicator. A network of additional computers performed the GPS navigation calculations, created the displays, altitude dependent wind and collected data. Wind was always a $45^{\circ}$ left or right head wind with respect to the final approach heading, but varied in strength using a power law atmospheric model. Pilots knew the wind direction varied, but were not told that only two relative wind directions were used. Since GPS system errors were not simulated, XTE was a direct measure of "Flight Technical Error" (FTE). 
Six current, multiengine, instrument rated pilots (750-3387 hrs. experience), were recruited locally. Each pilot flew 20 approaches, four with each display format over two tenapproach sessions. Eight approach charts were used, each with a different final approach heading and altitudes. Half the approaches were based on a GPS "T" approach geometry. The pilot was required to intercept the initial approach leg, and fly five miles to an intermediate approach waypoint (IF) at the
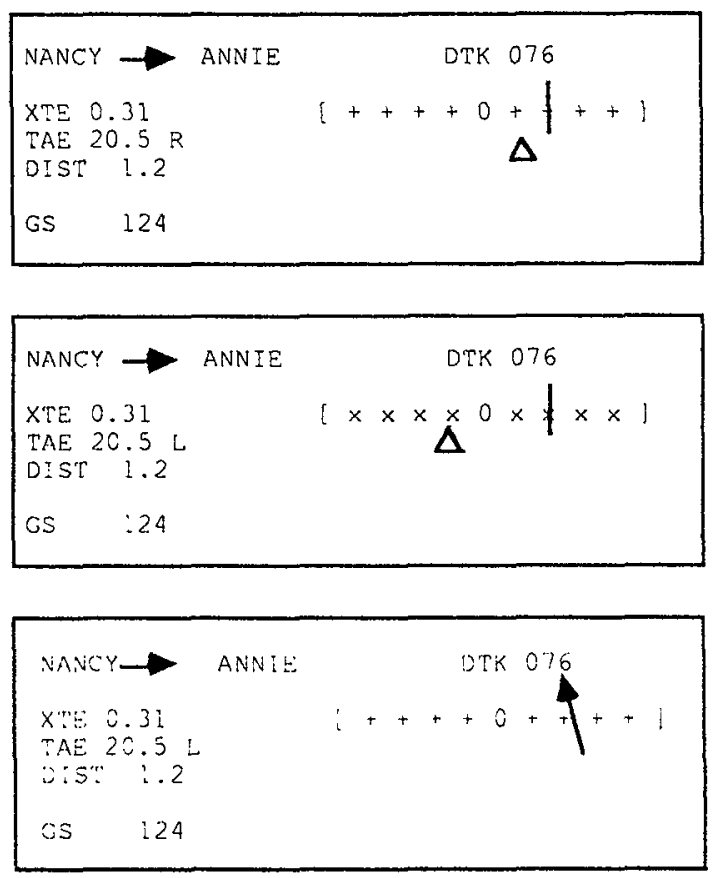

Fig. 4: (Top): Triangle/Same; (Middle):

Triangle/Opposite; (Bottom): Track Vector.

center of the " $\mathrm{T}$ ", maintaining $3100 \mathrm{ft}$. above ground level (AGL). At the IF, the pilot turned $90^{\circ}$ right or left, and flew five miles to the final approach fix (FAF). XTE display scale sensitivity changed from $\pm 1 \mathrm{~nm}$ to $\pm 0.3 \mathrm{~nm}$ at a waypoint 2 miles before the FAF. At the FAF, the pilot began descending to the $750 \mathrm{ft}$. minimum descent altitude (MDA), and flew five miles to the missed approach point (MAP) at the bottom of the T. At the MAP, the pilot then executed the missed approach procedure, and flew to the Missed Approach Holding Fix (MAHF). The remaining approaches used a more difficult "Crooked T" geometry in which the pilot was required to make a $45^{\circ}$ turn at the FAF, and then fly a descending, two mile dogleg before turning back to the runway heading. Pilots rated overall workload on a modified Bedford workload scale (Roscoe and Ellis, 1990; Huntley, et al 1993) after each approach. Al the end of the second session, pilots ranked the 5 displays on three preference scales: ease of interpretation (EOI), cffect on flight path control accuracy (FPA), and overall preference (OP). Also, they indicated the strength of their preference in "head to head" (HTH) comparisons of pairs of displays on $a \pm 7$ point analog scalc. The scores from the 10 pairs were summed and ranked using a tournament method, yielding a second measure of overall preference.

Ground tracks from each approach were normalized by rotation to a common final approach heading, leftright reversal where appropriate, and then compared by subject and display. The combined track records were used to retrospectively separate the approach into a series of 13 segments of varying lengths, chosen to isolate the various intercept, tracking, turning, descending and climbing phases of the approach. The mean, standard deviation, and RMS values of XTE, TAE, altitude, pitch and roll attitude along each segment were computed, and analyzed using Systat v.5.2. In addition, XTE was sampled at half mile intervals and averaged by display format. Mean and $95 \%$ limits of the XTE distribution were estimated based on the sample variance at each slice, using a method originally proposed by Huntley (1993). Differences in tracking performance, as measured by the variance of XTE for different pairs of displays were assessed based on their $F$ ratio.

\section{RESULTS}

In debriefing evaluations, pilots said that, as we anticipated, they were able to choose an intercept TAE, and then reduce it in several steps as they approached centerline. While tracking along a leg, an offset of the triangle or a tilt of the vector allowed pilots to detect and anticipate the magnitude and direction of slow changes in XTE. It was possible to immediately determine the cross wind correction angle without a cut-and-try approach. They learned to distinguish the "diverging" and "converging" XTE/TAE pointer configurations at a glance and react appropriately. If the XTE indicator was off scale, an appropriate indication on the TAE pointer reassured the pilot that XTE would soon be on scale again. The track vector display could be visualized as a track up moving map, but some pilots occasionally had difficulty maintaining the map interpretation, particularly immediately after waypoint changeover following a $90^{\circ}$ turn. There is reason to think performance with this format could be improved by making it appear more map-like and adding a vertical reference mark to permit vernier judgments of tilt. As shown in Table 1, questionnaire results

Table 1. Pilot Display Preference Ranks by display, using 4 different scales (see text). Rank $=1$ is best.

\begin{tabular}{lcccc}
\hline Display & \multicolumn{4}{c}{ Display Preference Scales } \\
Format & OP & HTH & FPA & EOI \\
\hline$\Delta /$ Same & 2 & 2 & 1 & 2 \\
$\Delta$ Opposite & 3 & 4 & 2 & 5 \\
Vector & 4 & 3 & 4 & 3 \\
HSI & 1 & 1 & 3 & 1 \\
XTE only & 5 & 5 & 5 & 4 \\
\hline
\end{tabular}

underscored the relative importance of having XTE in the primary instrument scan area, rather than alone on the GPS receiver. By a small numerical margin, pilots preferred the HSI display over the "triangle/same" TAE display on the OP and HTH scales. They preferred the triangle/same display over either of the other two TAE formats. All pilots always ranked one or more of the TAE display formats above the XTE only format on both scales, so the consensus was that TAE information was subjectively useful. (Four pilots preferred the "triangle/same" in direct comparison to the "triangle/opposite" format, and only one preferred the latter. ) In terms of FPA, piloss preferred the triangle/same display, though the three 


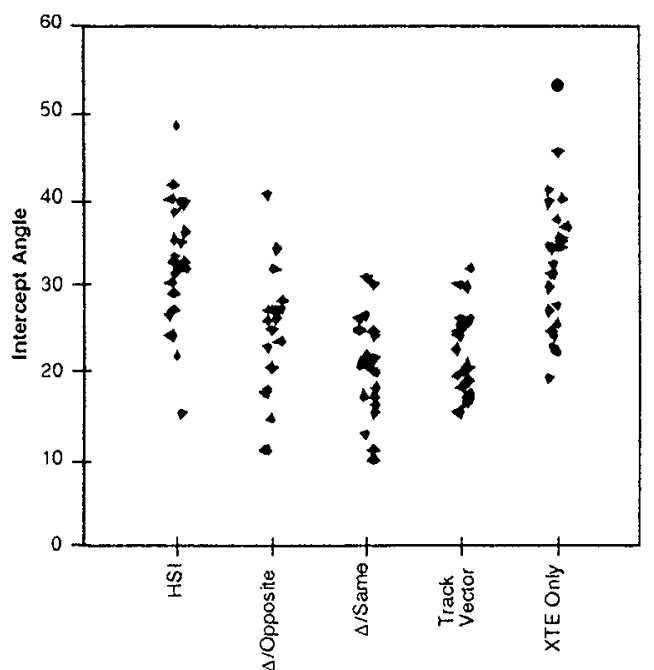

Fig. 5: Intercept Angle (degrees) vs. Display Type

subjects who actually tracked most consistently ranked the HSI first. Four of the five pilots said the HSI was the easiest of the displays to interpret, though three of the four cited longstanding training and experience with the HSI format as a reason for this. A significant effect of display was found for the HTH, FPA and EOI scale rank scores from the individual subjects (Friedman rank ANOVAs, $p<0.04$ ). For the OP scale results, the display effect was at the $p<0.06$ level. Four of the six pilots said they never referred to the numeric TAE information at all, since it was not easy to remember the meaning of the L/R TAE indication, and because the redundant analog TAE pointer was available. The $L$ and $R$ symbols were intended to indicate the direction of TAE.

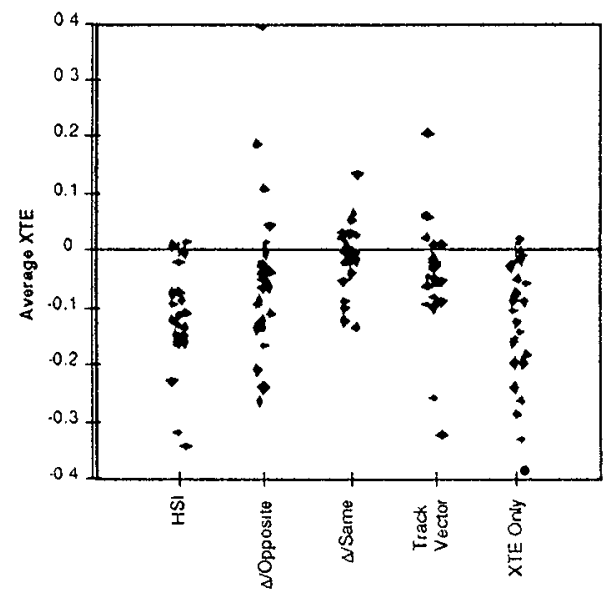

Fig. 6: Average XTE (nm) for miles 2 - 4 of Initial Approach Leg, vs. Display Type.

ANOVA of modified Bedford Workload scores revealed significant differences between subjects $(F(5,108)=29 ; p<0.0001)$, and $T$ and Crooked $T$ approach types $(F(1,108)=28.4 ; p<0.0001)$. However, adding display to the ANOVA did not reveal a significant effect. No trends were found by sequential approach or session number, suggesting training had asymptoted practice effects. Ranking workload scores within subjects did not reveal a display dependent effect. It was concluded that although the workloaci metric was demonstrably sensitive to approabhtwrgedomentrify.sagepub.com at DOTNOLPE CENTER on March 13, 2015 display effects, if they exist, were small compared to geometry and inter subject effects.

When using the TAE displays, pilots intercepted the initial leg of the approach and flew along it differently. We defined two intercept measures: 1) "Intercept Angle", the absolute value of TAE when XTE equaled $0.3 \mathrm{~nm}$ and 2) "Intercept Distance", the distance where the aircraft first crossed within $0.05 \mathrm{~nm}$ of the initial approach centerline. Intercept Angle (Fig.5) and Distance were consistently steeper and shorter, respectively, for approaches made with the nonTAE displays. The average increase in intercept angle with non-TAE formats approximated the drift angle to be expected (12 deg.) for pilots unaware of the crosswind. ANOVA of intercept angle data showed significant effects by display $(\mathrm{F}(4,85)=24.1, \mathrm{p}<0.0001)$, subject $(\mathrm{F}(5,85)=4.6$, $\mathrm{p}<0.001)$, and subject by display $(\mathrm{F}(20,85)=1.7, \mathrm{p}<0.04)$. The significant subject by display interaction effects indicate that some subjects used the TAE information differently than others. Contrasts comparing TAE and non-TAE intercept angle and distance results by display were both significant $(\mathrm{p}<$ $0.0001)$.

Once established on the initial leg, pilots flying with the triangle/same and vector displays had smaller downwind biases, as shown in Fig. 6 . The difference in average tracking bias was significant by display $(F(4,90)=7.8, p<0.001)$ and subject. $(F(5,90)=6.4 ; p<0.001)$. The subject by display interaction was not significant.

In terms of tracking performance during the initial, intermediate, and second missed approach, the HSI or Triangle/same displays generally ranked best, followed by the track vector display. Average display effects were smaller than intersubject effects. Inter subject differences in XTE and TAE tracking performance correlated with inner loop attitude control for the corresponding segments.

The 95\% XTE envelopes were calculated for all approaches and compared by display. An example is shown in Fig. 7. TAE display envelope widths were significantly different during portions of the approach. On the initial approach leg, $F$ ratio tests showed the triangle/same display XTE envelope was significantly narrower than for all other displays, and the centers of the initial approach envelopes for the HSI and XTE only formats were clearly displaced downwind, as compared to all three TAE based displays. During and immediately after the $90^{\circ} \mathrm{IF}$ turn, pilots using the HSI had significantly narrower XTE envelopes than with the other displays, by $\mathrm{F}$ ratio test. The track vector display envelope remained wide between the IF and the sensitivity changeover waypoint. Thereafter, XTE rapidly converged.

On $T$ geometry final approaches, the track vector display envelope was narrower than for any of the other displays. The differences disappeared at the MAP itself, perhaps because the pilots had shifted their attention to missed approach activities. Average $95 \%$ XTE envelope width during the last three miles of final approach provides a useful metric for $T$ approach performance comparisons. Average envelope widths were: HSI: $0.17 \mathrm{~nm}$, Triangle/opposite: $0.22 \mathrm{~nm}$, Triangle/same: $0.21 \mathrm{~nm}$, Track vector: $0.15 \mathrm{~nm}$, and XTE only: $0.23 \mathrm{~nm}$. Comparing the track vector result with that of XTE only, deletion of TAE vector information from the receiver display resulted in a 53\% increase in the average envelope width. 
On the Crooked $\mathrm{T}$ approaches, the envelopes consistently widened after the $45^{\circ}$ dogleg turns. However, reintercept performance and short final tracking was better with the track vector display. The track vector was the only one of the five displays for which the $\mathrm{CDI}$ is predicted to remain on scale through both turns in $95 \%$ of approaches flown. Next best performance was with the triangle/same display. The relatively poor performance with XTE only and HSI displays after $45^{\circ}$ turns using high CDI sensitivity suggests clear advantages for track vector and triangle/same displays when circumstances compel pilots to maneuver during the critical final stages of an instrument approach.
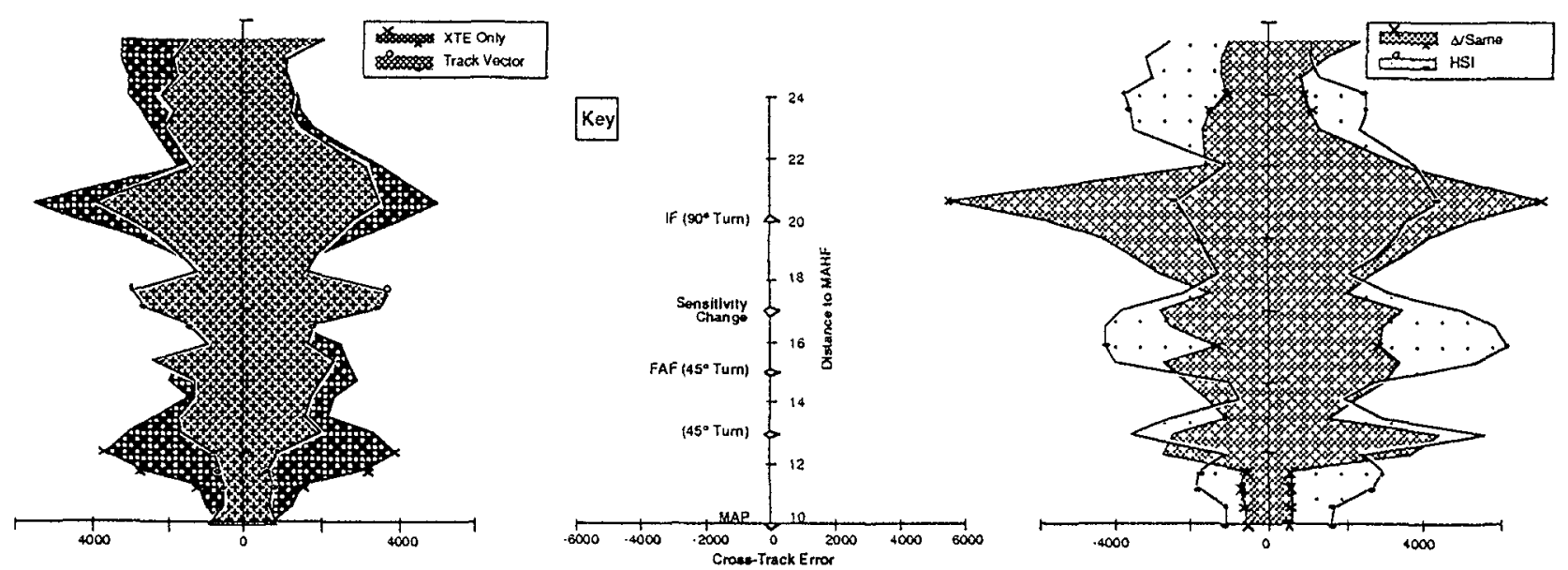

Fig. 7: Estimated 95\% limits of XTE distribution for all approaches combined. (Left): XTE only vs. Track Vector. (Right): Triangle/Same vs. HSI. (Middle): Key. Ordinates are distance from MAHF along the desired track. Circles and crosses denote slices were an $\mathrm{F}$ ratio test indicated a significant difference.

\section{CONCLUSIONS}

Results showed that under turbulence conditions requiring diligent attitude instrument scan, addition of analog TAE information to the receiver XTE display significantly improved approach intercept and tracking performance, probably by allowing the pilot to predict XTE changes and create outer loop control lead. Determination of wind correction angle was simplified. Pilots elected to use analog rather than numeric TAE. The "rriangle/same" TAE display produced the largest initial leg intercept and tracking improvement, and was preferred overall for flight path control. The "track vector" display produced the greatest reduction (35\%) in XTE envelope width during the last three miles of T approaches, as compared to the XTE only display. Control tests with a HSI showed the improvement due to addition of TAE to the receiver display is offset by the need to widen the pilot's instrument scan. It is likely that better performance be obtained by simultaneously displaying XTE on the HSI and XTE/TAE information on the GPS receiver. Although Bedford workload scores were sensitive to approach geometry, no consistent effect of display format on workload was found. Pilots may have chosen to keep workload constant, and allowed performance to vary instead. Overall, our findings support the FAA TSO-C129 recommendation that manufacturers provide analog TAE display capability and the requirement that analog XTE appear in the primary field of view. An experiment with 12 subjects comparing numeric TAE, analog TAE and XTE predictor displays is underway.

\section{ACKNOWLEDGMENTS}

We thank G. Lyddane, R. Disario, D. Hannon, T. CarpenterSmith, J. Bastow, S. Robinson, F. Shcelen, J. Giurlco, and J.
Turner, and also our subjects. The human use protocol was approved by committees at MIT and DOT/Volpe. Supported by MIT CTS Contract DTRS-57-92-C-0054 TTD\#27A and C.

\section{REFERENCES}

Clement, W.F., Jex, H.R., Graham, D. (1968). A Manual Control-Display Theory Applied to Instrument Landings of a Jet Transport. IEEE Trans. Man Machine Sys. 9:4 93-110

FAA (1994) Technical Standard Order C129:Airborne Supplemental Navigation Equipment Using the GPS. FAA, Washington, DC.

Etkin, B. (1980) The turbulent wind and its effect on flight. AIAA Aircraft Systems Meeting, Anaheim, CA, AIAA-80-1836.

Huntley, M.S. Jr. (1993) Flight Technical Error for Category B Non-Precision Approaches and Missed Approaches Using Non-Differential GPS for Course Guidance. Report DOT-VNTSC-FAA-93-17

Jansen, C.J. (1981) Non-Gaussian Atmospheric Turbulence Model for Flight Simulator Research. AIAA J. Aircraft 19 (1981): 374-379.

Oman, C.M., Huntley, M.S., Rasmussen, S.A.M., and Robinson S.K. (1995). The use of analog track angle error displays for improving simulated GPS approach performance. DOT/FAA report AR-95/104 US Department of Transportation, Federal Aviation Administration.

Roscoe, A.H. and Ellis, G.A., (1990) A subjective rating scale for assessing pilot workload in flight. Tech. Report TR90019, RAE Farnborough, UK.

RTCA Special Committee 15. (1991) Minimum Operational Performance Standards For Airbourne Supplemental Navigation Equipment Using Global Positioning System, DO-208 July, 1991, 1140 Connecticut Avenue, N.W., Washington, DC. 